\title{
Aplicación en formación de la realidad aumentada para el estudio de los huesos del cráneo utilizando dispositivos móviles
}

Application in formation of augmented reality for the study of skull bones using mobile devices

Aplicação na formação de realidade aumentada para o estudo de ossos do crânio usando dispositivos móveis

\section{Joffre Paladines Rodríguez}

Magister. Universidad Católica de Santiago de Guayaquil, Guayaquil Ecuador, joffre.paladines@cu.ucsg.edu.ec, https://orcid.org/0000-0001-9236-5135

\section{Daniel Nicolás Tettamanti Montalván}

Ing. Producción y Dirección en Artes Multimedia, Universidad Católica de Santiago de Guayaquil, Guayaquil Ecuador, dontetta@gmail.com, https://orcid.org/0000-0001-6910-5351

Recibido: 5 marzo 2017 - Aceptado 3 julio 2017

Formación docente - revista iberoamericana de educación http://www.revista-iberoamericana.org/index.php/es/index https://creativecommons.org/licenses/by/4.0/deed.es e-ISSN: 2737-632X

Vol - 1 No. 1, enero- diciembre 2018

Pags 94-109

Resumen Cranium es una aplicación móvil que utiliza la realidad aumentada, que sirve como un medio de apoyo para el estudiante de medicina para el estudio de los huesos del cráneo. El objetivo principal de la aplicación que sea utilizado en conjunto con el libro de Anatomía Humana - Latarjet, mediante el reconocimiento de imágenes presentes que se mostrarán en contenido aumentado y en 3D con sus elementos. Para lo cual se identifica métodos y técnicas de aprendizaje que se implementarán para el uso de la aplicación móvil. En la actualidad para los estudiantes de medicina resulta muy complicado tener acceso a los huesos en físico, ya que los recursos dentro de la facultad de medicina para estas clases de anatomía son muy limitado o escaso. Se plantea una investigación cualitativa, divida en tres fases: de identificación, de desarrollo de aplicación aumentada y de 
evaluación con los usuarios de la aplicación desarrollada, para lo cual se utiliza encuestas y entrevistas a profundidad para la recolección de información. Cranium busca mejorar esta situación a la vez de elevar el interés por la asignatura en los estudiantes sin sustituir al libro mencionado. Además, presentara preguntas de autoevaluación que abarcara los huesos estudiados ayudando a reforzar los conocimientos adquiridos. Todo esto fue obtenido a través de resultados de encuestas, entrevistas y pruebas realizadas con los estudiantes.

Palabras clave: Realidad Aumentada, Aplicación Móvil, Modelos 3D, Tecnología, huesos del cráneo

Abstract. Cranium is a mobile application that uses augmented reality, which serves as a means of support for the medical student to study the bones of the skull. The main objective of the application is to be used in conjunction with the book of Human Anatomy - Latarjet, using the recognition of images present in the book the augmented content will be shown in 3D with its elements. Currently for medical students, access to bones in physics is very complicated, since the resources within the school of medicine for these anatomy classes are very limited or scarce. Cranium seeks to improve this situation while raising interest in the subject in students without replacing the mentioned book. In addition, it will present self-assessment questions that will cover the bones studied, helping to reinforce the subject. All this was obtained through the results of surveys, interviews and tests carried out with the students.

Keywords: Augmented Reality, Mobile Application, 3D Models, Technology, skull bones 
Resumo. O Cranium é um aplicativo móvel que usa realidade aumentada, que serve como um meio de suporte para o estudante de medicina estudar os ossos do crânio. O principal objetivo do aplicativo é ser utilizado em conjunto com o livro de Anatomia Humana - Latarjet, utilizando o reconhecimento de imagens presentes no livro, o conteúdo aumentado será mostrado em $3 \mathrm{D}$ com seus elementos. Atualmente, para estudantes de medicina, o acesso aos ossos na física é muito complicado, pois os recursos da escola de medicina para essas aulas de anatomia são muito limitados ou escassos. O Cranium procura melhorar essa situação enquanto aumenta o interesse pelo assunto nos alunos sem substituir o livro mencionado. Além disso, apresentará questões de autoavaliação que cobrirão os ossos estudados, ajudando a reforçar o assunto. Tudo isso foi obtido através dos resultados de pesquisas, entrevistas e testes realizados com os alunos.

Palavras-chave: Realidade Aumentada, Aplicação Móvel, Modelos 3D, Tecnologia, ossos do crânio

\section{INTRODUCCIÓN}

Es objetivo es plantear una alternativa de estudio para los huesos del cráneo con el uso de la tecnología de la Realidad Aumentada de esta forma ofrecer algo innovador y entretenido. La adquisición de los datos ayudara al proyecto a cumplir su meta.

La formación de un profesional comienza desde su primer día como universitario y con los elementos de estudios básicos para su desarrollo como futuro profesional médico, La Universidad Católica de Santiago de Guayaquil, se encuentra en vías de desarrollo con respecto a estas nuevas formas y métodos de estudio para los estudiantes de la Facultad de Ciencias Médicas. Los estudiantes de medicina, para el aprendizaje de anatomía recurren a métodos tradicionales de estudio, libros, cuadernos con sus 
anotaciones, Internet, huesos humanos proporcionados por la asociación de estudiantes y cadáveres en la sala de disección dentro de la facultad.

La medicina y la tecnología siempre han ido de la mano, avanzando mutuamente y perfeccionándose. La medicina desde sus inicios se ha vuelto una parte esencial del ser humano, y la tecnología no se queda atrás, con cada avance de esta nos lleva un paso más adelante hacia una vida más cómoda y sencilla en todos los aspectos. Así también la formación que se brinda a los estudiantes de medicina debe de ser la mejor en todos los aspectos y gracias a los nuevos aportes tecnológicos se pueden logran cosas que uno solo soñaba hace más de 10 años.

La Facultad de Ciencias Médicas de la Universidad de Guayaquil admite cientos de estudiantes para su formación académica como profesionales de la medicina. El Dr. José Aguirre Amat, Decano de la Facultad de ciencias Médicas de la Universidad Católica de Santiago de Guayaquil, le resulta increíble como desde el dispositivo móvil uno puede apreciar las maravillas del ser humano con tan solo una aplicación quien indica que existen otros tipos de métodos además de los mencionados que los estudiantes pueden aprovechar para mejorar su rendimiento, tales como atlas interactivos online, aplicaciones móviles, incluso videojuegos educativos.

Este proyecto resalta los principales conceptos de la Realidad Aumentada, su aplicación en el ámbito de la salud y los beneficios de la misma. Por lo cual es necesario la creación de una aplicación que implemente la Realidad Aumentada que vaya orientada al estudio de los huesos del cráneo como una contribución tecnológica e informativa al entorno educativo de la Universidad Católica de Santiago de Guayaquil.

Los Smartphone forman parte de nuestra vida ya como un medio necesario de comunicación que interacciona con el resto de las personas en el mundo, pero en el ámbito educativo también posee sus usos como un apoyo para 
estudiar. De acuerdo con la INEC (Instituto Nacional de Estadísticas y Censos), afirmo en el 2015 que los usuarios de smartphones llegaron a más de 3 millones, y estos valores se elevaron casi cinco veces que lo reportado en el 2011 cuando la cifra llegaba a 500 mil personas. Además, en el 2017 concluyo que un $46.4 \%$ de usuarios del Servicio Móvil Avanzado poseen un smartphone.

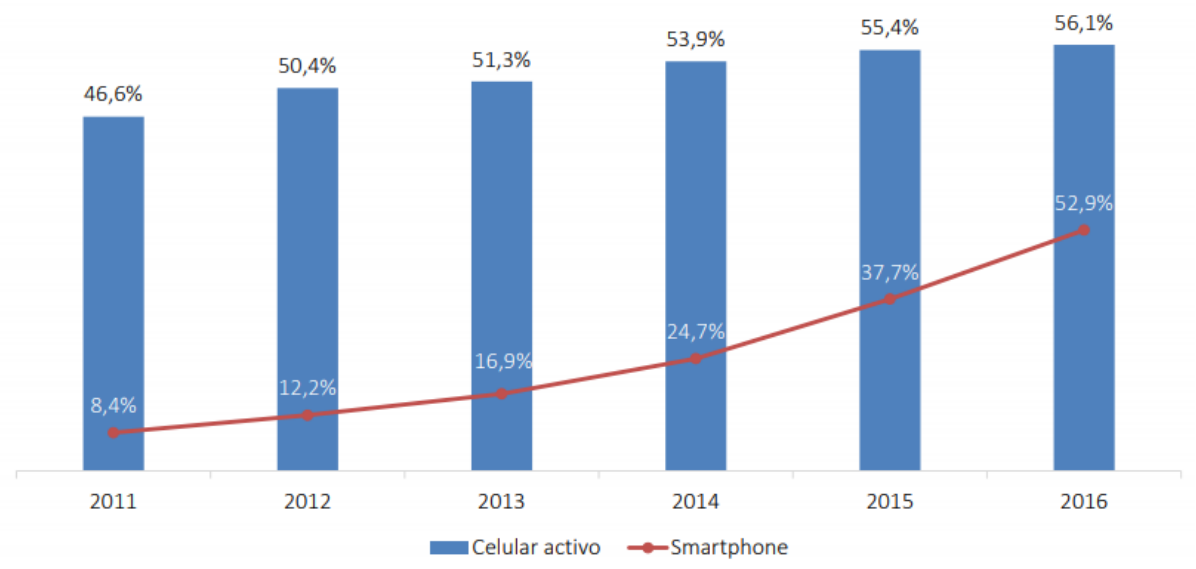

Figura 1. Encuesta de uso de Smartphones en el Ecuador. Fuente: Encuesta Nacional de Empleo Desempleo y Subempleo - ENEMDU (2014 - 2016)

Hoy en día las tecnologías para la educación se van desarrollando y mejorando en el transcurso del tiempo, y en el ámbito de la medicina es donde se ven con mayor frecuencia y apoyadas en estas nuevas tecnologías. Las Nuevas Tecnologías de la Información y Comunicaciones (TICs) proveen nuevas formas de estudio para los estudiantes de medicina en distintos países alrededor del mundo, Existen aplicaciones para casi todo lo que se puede imaginar, y las aplicaciones educativas no se quedan atrás, desde atlas interactivos, traductores, videos educativos, juego, entre otros; todos con el fin de educar o proveer una facilidad para mejorar el proceso de aprendizaje en las diversas asignaturas. 
Dentro del Plan Nacional para el Buen Vivir, se define a la educación como "un derecho de todas las personas y una obligación ineludible del Estado" (Garcés, s. f.) y además establecen estrategias para el mejoramiento del sistema educativo desde nivel primario hasta educación superior y como la implementación de las Tecnologías de la Información conllevan a un aporte positivo para este crecimiento como nación.

"La educación hace hoy la diferencia porque su doble función apoya los dos requerimientos importantes de la futura sociedad: el conocimiento resguarda la competitividad; la equidad resguarda la integración.” (Aguerrondo, 1999). La formación profesional de un médico debe de ser de la más alta calidad posible, ya que hablamos profesionales de la salud que lucharan día a día para salvar la vida de sus pacientes, y así mismo día a día se debe de mejorar la formación académica de los mismo.

En la Universidad Juárez Autónoma de Tabasco en México utilizan “Google Groups" un servicio gratuito de Google en el que permite al usuario crear foros de discusión, permite interactuar entre los participantes del grupo mediante texto, videos, imágenes, documentos o enlaces externos de forma pública o privada, en cambio La Universidad Católica Argentina y la Universidad Nacional del Nordeste en Argentina, utilizan mesas virtuales de anatomía, que permiten a los estudiantes de medicina realicen diversas variedades de disecciones y autopsias sobre cuerpos digitalizados, basados en estudios clínicos reales, en este sentido, específicamente en la Universidad de Especialidades Espíritu Santo y la Universidad Estatal de Guayaquil, han incorporado el Anatomage, que es una mesa de disección virtual para el estudio de la anatomía humana y diagnóstico clínico.

Un estudio realizado en la Universidad Pablo de Olavide, de Sevilla en España concluyo: "que las actividades didácticas con base en la RA pueden ser especialmente útiles en estudios preuniversitarios y de enseñanza superior 
relacionados con las áreas de Humanidades y Ciencias Sociales ya que permite un acceso a contenidos, muchas veces presentados de forma más unidireccional mediante medios impresos que no incrementan la motivación o requieren un mayor esfuerzo de abstracción entre los estudiantes" (CaberoAlmenara, Vázquez-Cano, \& López-Meneses, 2018); entonces es innegable que las tecnologías influyen de manera positiva en el rendimiento de los estudiantes.

La Facultad de Ciencias Médicas de la Universidad Católica de Santiago de Guayaquil, para impartir clases de Anatomía utilizan las salas de disección, atlas 3D que los Docentes poseen y huesos donados por la Asociación de Estudiantes, mientras otras universidades del país están implementando nuevas tecnologías desde los primeros ciclos o semestres de estudio, existe una cantidad de instituciones universitarias que no tienen la posibilidad de implementar nuevas tecnologías o de invertir en algún tipo de tecnológica que aporte positivamente al desarrollo estudiantil y solo les es posible llegar a obtener computadoras que conforme avance el tiempo deberán ser reemplazadas por versiones más actualizadas.

Centrándonos en el área educativa, la utilización de las Tecnologías de la Información y Comunicación (TICs) inicio a la introducción de las famosas pizarras digitales, y poco a poco estas tecnologías han ido evolucionando creando las denominadas Tecnologías Emergente cuya didáctica potencian la enseñanza.

Importancia de la Realidad Aumentada en la Educación

En el sector educativo la realidad aumentada está tomando fuerza debido a su capacidad de añadir información virtual a un mundo físico, en el que permite al estudiante captar la información de una manera más interactiva y visual, aumentando el interés por el estudio. 
A medida que la informática va avanzando, estas mismas tecnologías llegan a cruzarse con la Realidad Aumentada es por ello que (Zheng, Zhang, \& Yang, 2015) han dividido las aplicaciones que utilizan Realidad Aumentada en 3 categorías: Adquisición de datos en entorno real, registro de objetos virtuales en el entorno real y presentación de datos compuestos, independientemente de la tecnología empleada.

Al hablar de Realidad Aumentada (Augmented Reality) (RA), nos centramos en una de las tecnologías a las cuales se las denomina Tecnología Emergente que cada vez y con más fuerza se está acercando a diferentes terrenos de la sociedad, ya sea el ocio, publicidad, marketing, entretenimiento y por supuesto el ámbito educativo como herramienta de apoyo tanto para docentes y estudiantes.

La Realidad Aumentada tiene sus inicios en 1950 por Morton Heilig, quien buscaba alguna forma que el espectador pudiera incorporar de manera efectiva todos sus sentidos en la pantalla. Es así que construyo un prototipo llamado Sensorama en 1962 junto con cinco filmes cortos que buscaba aumentar la experiencia del espectador. (Heilig, 1971). "La Realidad Aumentada es una tecnología que aumenta la percepción sensorial humana con información auxiliar, que puede mejorar potencialmente el rendimiento en la realización de una tarea o experiencia" (Caudell \& Mizell, 1992)

La Realidad aumentada es la tecnología que nos permite superponer/agregar elementos o datos virtuales (texto, hiperenlaces, audio, video, multimedia, entre otros.) a la realidad existente a partir de un objeto del mundo real, en lugar de crearlas desde cero. Para ello se necesita un dispositivo ya sea móvil, Tablet o portátil, con una cama y software que procesa la información, activadores de realidad aumentada y una pantalla donde mostrar la imagen real. 
Las grandes empresas tecnológicas saben que esta realidad promete mucho en el aspecto económico, y se encuentran desarrollando gran cantidad de aplicaciones y gadgets que invitan al usuario a invertir en esta nueva experiencia. Google lo logra con sus gafas Daydream View, Sony con "Eye Toy" un juego desarrollado en 2003, y Niantic al crear "Pokemon GO”, y así muchas empresas como Samsung, Snap Inc y Apple están implementado esta tecnología.

Al hablar de realidad aumentada varios autores (Rice, 2009) han establecidos que una clasificación de la realidad aumentada como tal debe efectuarse en niveles, entendiéndose que, a mayor nivel, mayor serán las posibilidades de las aplicaciones tales como: nivel 0 que hace referencia a hiperenlaces en el mundo físico, nivell hace referencia a realidad aumentada en marcadores, nivel 2 utiliza realidad aumentada sin marcadores,nivel3 utiliza visión aumentada

La realidad aumentada ofrece una infinidad de posibilidades de interacción tanto en el entretenimiento, arquitectura, arte, comunidades virtuales o la medicina, "Chromville" creada en el 2011 y lanzado en el 2014 en Zaragosa, España es una aplicación sencilla creada por la empresa "Imascono" dirigida a niños de entre 5 a 12 años, con fichas predefinidas las cuales los niños colorean y al utilizar la aplicación veremos cómo estos dibujos cobran vida en animaciones 3D. "El mundo educativo de Chromville está representado en una app infantil de Realidad Aumentada. Su objetivo, fomentar el desarrollo de la imaginación y la creatividad de los niños.” («App infantil con Realidad Aumentada, el universo Chromville», s. f.).

"Quiver" aplicación infantil creada por "QuiverVision” la cual permite a los niños tener una experiencia nueva con el mundo y su creatividad, con tan solo imprimir y colorear las imágenes prediseñadas por la compañía y al utilizar la aplicación sus creaciones cobran vida en el celular en tiempo real. "Nuestra 
misión es producir contenido educativo que soporte el aprendizaje de forma divertida y estimulante («Home - Quiver 3D Augmented Reality coloring apps», 2018)

“Anatomy 4D" es una aplicación de realidad aumentada utilizada para el estudio de la anatomía del cuerpo humano. Creada por "DAQRI" esta aplicación utiliza modelos 3D que se muestran sobre las plantillas predefinidas por la compañía que deben ser impresas para el uso debido de la aplicación. "Este entorno de aprendizaje tridimensional y fácil de usar es ideal para usarlo en el aula o en cualquier momento".(Ltd, 2018).

También se está implementando para mejorar la experiencia de los museos, ya sea para lograr ver piezas antiguas de la historia humana que han sido desgastadas o destruidas por el tiempo de forma completa, o crear una experiencia de inmersión con el usuario, como lo fue realizado en "Una Experiencia Glaciar" en Argentina donde el usuario puede observar cómo funciona la represa de Yacycretá.

En España, la asociación "Espiral" conformado por personas con una formación técnica, investigadores, estudiantes y entidades crearon el movimiento "Auméntame" con una misión, llevar las últimas tecnologías en la educación a distintas instituciones educativas, para mejorar la experiencia de los estudiantes en el aprendizaje y para la formación del profesorado para una mejor educación. («Realidad Aumentada y Virtual», 2018)

\section{MATERIALES Y MÉTODOS}

La presente investigación está enfocado a una investigación metodológica cualitativa, ya que se plantea realizar una solución a hechos y situaciones actuales de los estudiantes de la facultad de medicina de la Universidad Católica de Santiago de Guayaquil, dividiéndolo en tres fases:

- Fase de Identificación 
- Identificación de sistema operativo predominante.

- Catalogación de contenidos virtuales.

- Creación virtual de los elementos escogidos.

- Fase de desarrollo de la aplicación aumentada.

- Fase de evaluación con usuarios de la aplicación desarrollada

Para llevar a cabo el análisis se combinará estudios cuantitativos y cualitativos que determinaran los elementos claves para el desarrollo de la aplicación interactivo con realidad aumentada para la visualización de los huesos del cráneo utilizando el Libro de "Anatomía Humana - Latarjet 4ta edición" usado por los estudiantes de anatomía de la facultad de medicina. Se analizarán los datos de las encuestas para poder recolectar información para el desarrollo de la aplicación y tener distintos puntos de vistas tanto de los estudiantes como de los docentes. Además de una entrevista a profundidad que explicara si la introducción de la nueva tecnóloga al museo es o no es viable.

Robert Rice, incluso nos menciona un cuarto nivel, en el que la tecnología avanzara de tal manera que los dispositivos de realidad aumentada estarán conectados directamente a nuestros nervios ópticos y que nos adentramos en un mundo donde colisionaran diversas realidades y se mezclaran para crear una sola. "Aquí es donde la Realidad Virtual llegara a su fin y nuestros sueños de inmersión pura y total donde olvidaremos nuestros cuerpos finalmente se realizarán" (Rice, 2009)

Se utilizaron varios instrumentos para la recolección de información que permita sustentar y soportar la vialidad del desarrollo de la aplicación móvil, entre ellos tenemos encuesta y entrevista. Las encuestas fueron dirigidas a los estudiantes de la facultad de medicina y mediante sus respuestas tener un conocimiento del campo en el que se está trabajando y obtener datos desde la perspectiva del estudiante. La entrevista se utilizó para obtener información 
sobre la situación actual de la facultar, que innovaciones se han ido implementando en la materia de anatomía y cuáles fueron los puntos de vista de los docentes entrevistados.

Con la finalidad de presentar el proyecto en la actualidad y medir el impacto que tendría una aplicación educativa utilizando la realidad aumentada. La encuesta fue realizada a 169 estudiantes de primer ciclo de la facultad de medicina para conocer sus puntos de vista para el desarrollo de la aplicación. Partiendo de la población obtenida gracias a la Facultad de Medicina que consta de 300 estudiantes inscritos en primer ciclo de la carrera de Medicina se escogió el 56.4\% de la población. Se aplicó un nivel de confianza del 95\% definiendo como límite de error un 5\%.

\section{RESULTADOS}

La recolección de información requerida se encuesto a 169 estudiantes que cursan el primer ciclo de la carrera de medicina en la facultad de ciencias médicas. Estas encuestas fueron realizadas de forma presencial, se procedió con el análisis e interpretación de cada una de las preguntas dentro de la encuesta con el fin de obtener los puntos de vista de los estudiantes en cuanto a la utilización de las nuevas tecnologías para la educación, la variedad de elementos tecnológicos que los estudiantes utilizan para estudia. Con una notable inclinación hacia los videos de un 47\%. Luego están los atlas 3D representando un 29\% y las aplicaciones no quedan atrás ya que un 23\% afirman utilizarlas para estudiar.

los resultados de la encuesta es claro afirmar que de los 169 encuestados, la respuesta de "Muy Necesario" es de un 87\% el cual nos indica la gran necesidad de implementar nuevas tecnologías en la educación.

Se comprueba que los estudiantes utilizan libros y anotaciones para el estudio, un $47 \%$ de ellos también utilizan contenido multimedia para ayudarse en el proceso de aprendizaje en clase; representado por el 29\% utilizan Atlas 3D 
como un elemento más de aporte al estudio de la materia y sin dejar atrás a las aplicaciones, se obtiene un 23\% utilizan aplicaciones móviles educativas como otro elemento de estudio. Se comprueba que la mayoría de los encuestados, el 98\% afirmo de manera positiva la necesidad de tener una aplicación que utilice la realidad aumentada para el estudio de los huesos del cráneo en sus clases Anatomía.

\section{CONCLUSIONES}

Los resultados de la primera evaluación heurística arrojaron resultados satisfactorios, a pesar de que la aplicación se encuentra en la fase de prototipo; es decir solamente pantallas con contenido y transición entre ella.

A nivel del Icono de la aplicación fue muy bien calificada con solo una pequeña observación de modificarlo un poco su aspecto, pero su diseño va acorde al target y el diseño de la interfaz. El Menú es adaptativo en su totalidad y en la gran mayoría de las pantallas del prototipo va acorde al diseño de la aplicación, pero existe problemas en la forma que indica las acciones dentro de la aplicación. El mayor problema dentro del diseño de la aplicación es la tipografía utilizada, es muy pequeña en ciertas áreas y difícil de leer en su mayoría. Tomando en cuenta estos resultados se realizarán cambios que ayudarán a la aplicación a ser más agradable y funcional para los usuarios, Las encuestas determinaron que los estudiantes tienen conocimiento sobre esta tecnología y que existe un alto nivel de aceptación hacia la implementación de esta tecnología para el estudio de los huesos del cráneo.

Luego de los análisis se realizó el modelado de los huesos del cráneo, se elaboró los marcadores que serán usados por la cámara de realidad virtual y se creó la autoevaluación en base al texto utilizado por los estudiantes. 


\section{REFERENCIAS}

Aguerrondo, M. I. (1999). La trama de la escuela media. Atando y desatando nudos.

App infantil con Realidad Aumentada, el universo Chromville. (s. f.). Recuperado 29 de junio de 2018, de http://imascono.com/es/portfolio-item/app-infantil-chromville

Barros-Bastidas, C., \& Turpo, O. (2020). La formación en investigación y su incidencia en la producción científica del profesorado de educa- ción de una universidad pública de Ecuador. Publicaciones, 50(2), 167-185. doi:10.30827/publicaciones.v50i2.13952

Barros Bastidas, C., \& Turpo Gebera, O. (2018). Factors influencing the scientific production of university professors: a systematic review . Pensamiento Americano, 11(22).

https://doi.org/10.21803/pensam.v11i21-1.276

Cabero-Almenara, J., Vázquez-Cano, E., \& López-Meneses, E. (2018). Uso de la Realidad Aumentada como Recurso Didáctico en la Enseñanza Universitaria. Formación universitaria, 11(1), 25-34. https://doi.org/10.4067/S0718-50062018000100025

Caudell, T. P., \& Mizell, D. W. (1992). Augmented reality: an application of heads-up display technology to manual manufacturing processes (pp. 659-669 vol.2). IEEE. https://doi.org/10.1109/HICSS.1992.183317

Garcés, L. (s.f.). REPÚBLICA DEL ECUADOR CONSEJO NACIONAL DE PLANIFICACIÓN, Garcés, L. M - Buscar con Google. Recuperado 30 de agosto de 2018 , https://www.google.com.ec/search?q=REP\%C3\%9ABLICA+DEL+ECUADOR+CO NSEJO+NACIONAL+DE+PLANIFICACI\%C3\%93N\%2C+Garc\%C3\%A9s\%2C+L. $+\mathrm{M} \&$ oq $=\mathrm{REP} \% \mathrm{C} 3 \% 9 \mathrm{ABLICA}+\mathrm{DEL}+\mathrm{ECUADOR}+\mathrm{CONSEJO}+\mathrm{NACIONAL}+\mathrm{DE}+\mathrm{P}$ LANIFICACI $\%$ C3\%93N\%2C+Garc\%C3\%A9s\%2C+L.+M\&aqs=chrome..69i57.372 44j0j7\&sourceid $=$ chrome\&ie $=\mathrm{UTF}-8$ 
Heilig, M. L. (1971). Experience theater. United States. Recuperado de https://patents.google.com/patent/US3628829A/en

Home - Quiver 3D Augmented Reality coloring apps. (2018, junio 29). Recuperado 29 de junio de 2018, de http://www.quivervision.com/

Ltd, E. (2018, junio 29). Anatomy 4D Review. Recuperado 29 de junio de 2018, de https://www.educationalappstore.com/app/anatomy-4d

Realidad Aumentada y Virtual: Descubriendo sus posibilidades en Educación y Formación - Education \#EDUinsights. (2018, marzo 22). Recuperado 20 de junio de 2018, de http://aumenta.me/2018/03/22/realidad-aumentada-y-virtual-descubriendo-susposibilidades-en-educacion-y-formacion-education-eduinsights/

Rice, R. (2009, marzo). Augmented Vision and the Decade of Ubiquity. Recuperado 29 de junio de 2018, de http://www.curiousraven.com/future-vision/2009/3/20/augmentedvision-and-the-decade-of-ubiquity.html

von Feigenblatt, Otto Federico, ASEAN and Human Security: Challenges and Opportunities (July 29, 2009). Ritsumeikan Center for Asia Pacific Studies Working Paper, No. 095 , Available

SSRN: https://ssrn.com/abstract=1442476 or http://dx.doi.org/10.2139/ssrn.1442476 von Feigenblatt, Otto Federico, Japanese Animation as a Global Product: The Lingering Traces of Nijonjinron and the Rise of Globalism and Hybridity (August 2, 2012). Journal of History \& Social Sciences, 2(2), pp. 1-14, July-December 2012, Available at SSRN: $\underline{\text { https: } / / \text { ssrn.com/abstract }=2195562}$

von Feigenblatt, Otto Federico, Garcia Marquez' Magical Realism as a Representation of

Latin America's Socio-Political Reality: Developmental Simultaneity and Exceptionalism in Latin America as Expressed in Historiographic Metafiction (December 27, 2009). The Expression, Vol. 2, No. 1, pp. 1-6, 2009, Available at SSRN: $\underline{\text { https://ssrn.com/abstract }=1596690}$ 
Zheng, R., Zhang, D., \& Yang, G. (2015). Seam the Real with the Virtual:a Review of Augmented Reality. Atlantis Press. https://doi.org/10.2991/itoec-15.2015.17 